\title{
Genome assemblies of Fusarium oxysporum f. sp. lini strains of different virulence
}

\author{
Novakovskiy R.O. ${ }^{1 *}$, Dvorianinova E.M. ${ }^{1,2}$, Rozhmina T.A. ${ }^{3}$, Kudryavtseva L.P. ${ }^{3}$, \\ Povkhova L.V. ${ }^{1,2}$, Pushkova E.N. ${ }^{1}$, Melnikova N.V. ${ }^{1}$, Dmitriev A.A. ${ }^{1}$ \\ ${ }^{1}$ Engelhardt Institute of Molecular Biology, Russian Academy of Sciences, Moscow, Russia \\ ${ }^{2}$ Moscow Institute of Physics and Technology, Dolgoprudny, Russia \\ ${ }^{3}$ Federal Research Center for Bast Fiber Crops, Torzhok, Russia \\ * email: Olegovich46@mail.ru
}

Since the last century Fusarium oxysporum f. sp. lini has been known as one of the most devastating flax pathogens. At the same time, the severity of the disease symptoms it causes varies, whereas the forma specialis includes numerous strains of different pathogenicity degrees. However, the molecular basis of such diversity has not been investigated yet. To fill the knowledge gap, we performed whole-genome sequencing of 5 strains of $F$. oxysporum f. sp. lini of different pathogenicity degrees: high (\#483), medium (\#476, \#525), and low (\#456, \#482) ones. We obtained the DNA of these fungi according to our previously developed protocol and sequenced the libraries on Oxford Nanopore Technologies (MinION, R9.4.1, 10× coverage on average) and Illumina (MiSeq, $300+300 \mathrm{bp}$, 50x coverage on average) platforms. Using both types of the obtained raw data as well as the Nanopore and Illumina reads of a highly pathogenic isolate \#39, which were received earlier, we assembled the genomes of the 6 strains with a hybrid assembler MaSuRCA. The lengths of the assemblies were in the range of 53$69 \mathrm{Mb}, \mathrm{N} 50$ values $-1.0-2.5 \mathrm{Mb}$, and the BUSCO completeness - 96.5-99.7\%. Then, the sequences of the SIX genes of F. oxysporum f. sp. lini (SIX1, SIX7, SIX11, SIX12, SIX13) were downloaded from GenBank, as this class is associated with virulence in F. oxysporum species. The search with BLAST (the blastn algorithm) revealed that all our assemblies contain the query sequences except for the genome of the low virulent strain \#482. The received data helps understand the virulence mechanisms of the flax pathogen and elaborate methods to combat it.

Acknowledgements: This work was funded by RFBR according to the research project 19-34-90055. 